Revista Arbitrada Interdisciplinaria KOINONIA

Año 2020. Vol V. №3. Especial: Administración

Hecho el depósito de Ley: FA2016000010

ISSN: 2542-3088

FUNDACIÓN KOINONIA (F.K). Santa Ana de Coro. Venezuela.

Verónica Alexandra Bernal-Rodas; Moisés Marcelo Matovelle-Romo;

Cristina Guadalupe Ordoñez-Espinoza; Magdalena Emilia Ordoñez-Gavilanes

http://dx.doi.org/10.35381/r.k.v5i3.892

\title{
Gestión de calidad del capital humano en la Universidad Católica de Cuenca
}

Quality management of human capital at the Catholic University of Cuenca

\author{
Verónica Alexandra Bernal-Rodas \\ vbernalr@psg.ucacue.edu.ec \\ Universidad Católica de Cuenca, Cuenca \\ Ecuador \\ https://orcid.org/0000-0001-5381-6562 \\ Moisés Marcelo Matovelle-Romo \\ mmmatoveller@ucacue.edu.ec \\ Universidad Católica de Cuenca, Cuenca \\ Ecuador \\ https://orcid.org/0000-0002-6260-6644 \\ Cristina Guadalupe Ordoñez-Espinoza \\ cgordoneze@ucacue.edu.ec \\ Universidad Católica de Cuenca, Cuenca \\ Ecuador \\ https://orcid.org/0000-0001-5781-7781 \\ Magdalena Emilia Ordoñez-Gavilanes \\ meodonezg@ucacue.edu.ec \\ Universidad Católica de Cuenca, Cuenca \\ Ecuador \\ https://orcid.org/0000-0001-7860-1314
}

Recibido: 20 de mayo de 2020

Revisado: 15 de junio de 2020

Aprobado: 30 de julio de 2020

Publicado: 15 de agosto de 2020 


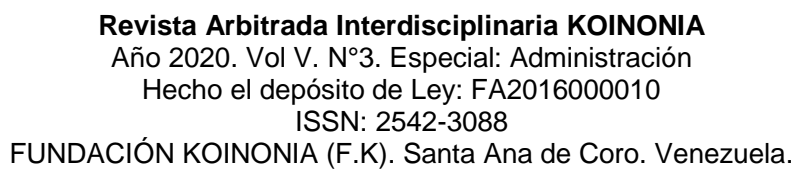

Verónica Alexandra Bernal-Rodas; Moisés Marcelo Matovelle-Romo;

Cristina Guadalupe Ordoñez-Espinoza; Magdalena Emilia Ordoñez-Gavilanes

\title{
RESUMEN
}

La investigación tiene por objetivo analizar la gestión de calidad del capital humano en la Universidad Católica de Cuenca. Metodológicamente se basó en un tipo descriptivo no experimental transversal, con la finalidad de recopilar información mediante encuesta online y cuestionario de varias alternativas de respuesta en escala de Likert, la muestra encuestada, estuvo constituida por 124 estudiantes. Con la información graficada y con los datos recolectados se puede analizar que el $60 \%$ de encuestados desconocen si la universidad cuenta con un sistema de gestión de calidad. Se recomienda la implementación de un sistema de gestión de calidad mismo que permitirá monitorear la planificación, control, mejoramiento y mejora continua de la calidad, para brindar un servicio de alta calidad.

Descriptores: Organización privada; satisfacción en el trabajo; servicio de oficina; gestión de documentos. (Palabras tomadas del Tesauro UNESCO).

\begin{abstract}
The research aims to analyze the quality management of human capital at the Universidad Católica de Cuenca. Methodologically, it was based on a descriptive, non-experimental, cross-sectional type, in order to collect information through an online survey and a questionnaire of various response alternatives on the Likert scale, the surveyed sample consisted of 124 students. With the graphical information and the collected data, it can be analyzed that $60 \%$ of respondents do not know if the university has a quality management system. The implementation of a quality management system itself is recommended that will allow monitoring the planning, control, improvement and continuous improvement of quality, to provide a high quality service.
\end{abstract}

Descriptors: Private organizations; job satisfaction; office services; records management. (Words taken from the UNESCO Thesaurus). 
Revista Arbitrada Interdisciplinaria KOINONIA

Año 2020. Vol V. №3. Especial: Administración

Hecho el depósito de Ley: FA2016000010

ISSN: 2542-3088

FUNDACIÓN KOINONIA (F.K). Santa Ana de Coro. Venezuela.

\begin{abstract}
Verónica Alexandra Bernal-Rodas; Moisés Marcelo Matovelle-Romo;
Cristina Guadalupe Ordoñez-Espinoza; Magdalena Emilia Ordoñez-Gavilanes
\end{abstract}

\title{
INTRODUCCIÓN
}

En el Ecuador la concepción sobre la calidad, que existe hoy en día en la educación superior, es el resultado de cambios internos y externos que afectan a las bases institucionales que han requerido un cambio en los procesos, lo cual se ha evidenciado fundamentalmente en los últimos 30 a 40 años. Cuando se habla de la gestión de calidad dentro de la Educación Superior, se debe considerar que eso nace de la necesidad de medir los impactos que se podrán obtener en los procesos de evaluación permitiendo así, una acreditación eficaz, siendo considerado por el Consejo de Aseguramiento de la Calidad de Educación Superior (CACES) como ejes fundamentales al momento de verificar los parámetros de eficiencia de las Instituciones de Educación Superior (IES).

Para medir la calidad educativa de una universidad, el punto clave es el proceso, es decir las fases y componentes de la actividad universitaria, los elementos que en ella intervienen; el resultado se enfoca en la misión y la visión de dicha institución, sí se logra en términos de formación de profesionales, de científicos, generando avances en la investigación y no menos importante, aunque más difícil de medir, los beneficios que se obtienen para la sociedad.

De acuerdo con lo determinado por la Norma ISO 9001:2015, la necesidad en cuanto a la adopción y el desarrollo de un Sistema en el cual se refleje claramente la Gestión de la Calidad, lo cual resulta una decisión estratégica que a su vez permite a las organizaciones mejorar continuamente su rendimiento general, centrándose en proporcionar productos y servicios de calidad a los clientes (Cuello-Cojitambo, et al., 2020). De ese modo, se aborda el análisis de los servicios administrativos, especialmente en el área de las secretarías de las Unidades Académicas de la Universidad Católica de Cuenca, donde se centran la gran parte de procesos, los cuales deben contar con un sistema que permita evaluar mediante gestión de calificación cuantitativa, la atención y celeridad de los funcionarios de estas dependencias. 
Revista Arbitrada Interdisciplinaria KOINONIA

Año 2020. Vol V. №3. Especial: Administración

Hecho el depósito de Ley: FA2016000010

ISSN: 2542-3088

FUNDACIÓN KOINONIA (F.K). Santa Ana de Coro. Venezuela.

\section{Verónica Alexandra Bernal-Rodas; Moisés Marcelo Matovelle-Romo; Cristina Guadalupe Ordoñez-Espinoza; Magdalena Emilia Ordoñez-Gavilanes}

Por eso es necesario que, dentro de las secretarías de las Unidades Académicas de la Universidad Católica de Cuenca, se creen procesos para que los estudiantes puedan evaluar el servicio brindado por los funcionarios que laboran en estas dependencias, lo cual permitirá identificar las fortalezas y debilidades que se dan en el desarrollo de los procesos que se realizan, a su vez tomar las medidas necesarias para mejorar el servicio brindado. Para lo antes expuesto la calidad se podría medir a través de la percepción y satisfacción de los estudiantes, de todos quienes requieran los servicios de las secretarías de las Unidades Académicas, en cuanto a procesos administrativos, lo cual podría evaluarse mediante un sistema tecnológico en el cual luego de efectuar dicho trámite califique objetivamente el servicio recibido.

Por lo anterior descrito, la investigación tiene por objetivo analizar la gestión de calidad del capital humano en la Universidad Católica de Cuenca.

\section{Referencial teórico}

\section{Expectativa, percepción y satisfacción del usuario o cliente}

En este sentido es necesario que evaluemos también que es la expectativa del cliente y lo podríamos definir como la esperanza de hacer o conseguir algo, o la posibilidad razonable de que algo suceda, pues la expectativa del usuario es también uno de los elementos fundamentales para la evaluación que se pretenda dar por parte de quienes denominamos los usuarios del bien o servicio, para poder concluir si este ha cumplido con las expectativas, en este sentido, la expectativa del cliente consiste en "las perspectivas o creencias sobre la entrega de servicios que sirven como estándares 0 puntos de referencia para juzgar el desempeño de la empresa" (Matsumoto-Nishizawa, 2014), así mismo, las principales expectativas, dimensiones y claves al momento de evaluar la calidad de un servicio, se debe tener en cuenta lo siguiente:

1. Empatía: está relacionada con la atención que un miembro de la organización o empresa brinde a sus clientes o usuarios. 
Revista Arbitrada Interdisciplinaria KOINONIA

Año 2020. Vol V. №3. Especial: Administración

Hecho el depósito de Ley: FA2016000010

ISSN: 2542-3088

FUNDACIÓN KOINONIA (F.K). Santa Ana de Coro. Venezuela.

\section{Verónica Alexandra Bernal-Rodas; Moisés Marcelo Matovelle-Romo; Cristina Guadalupe Ordoñez-Espinoza; Magdalena Emilia Ordoñez-Gavilanes}

2. Seguridad: podría afianzarse con el conocimiento y atención que muestra el miembro de la empresa al momento de ofrecer el servicio, es decir sus habilidades que deben inspirar confianza y credibilidad.

3. Capacidad de Respuesta: es la disposición y voluntad para brindar el servicio rápido a los clientes o usuarios.

4. Fiabilidad: se traduce en la confianza que se transmite a los usuarios de que se va a brindar con garantía el servicio que se ha ofertado.

5. Tangibles: está relacionado con el aspecto de las instalaciones de la organización o empresa, el equipamiento de esta y la apariencia del personal (Bernal-Ávila, et al, 2019).

La percepción del cliente o usuario se refiere al momento de recibir el producto o el servicio, se aprecia y posteriormente se valora, esto sin lugar a duda está directamente relacionado con las características del servicio, sin embargo, es necesario que se tenga en cuenta que esta percepción puede variar de acuerdo a las experiencias propias de cada usuario o cliente, las experiencias que tengan y los saberes previos. Esta percepción se forma cuando se evalúa el paquete de beneficios que le ofrece la empresa, determina la relación costo/beneficio y decide si la sensación es buena" (Caldera, et al., 2011).

En cuanto a la satisfacción del cliente debemos tener presente que es un requisito predominante para poder determinar la fidelidad que el cliente o usuario va a tener con la empresa o institución, es decir al momento que se tiene satisfecho al usuario podemos estar tranquilos por cuanto este factor repercutirá en los beneficios que va a tener la empresa, por lo tanto es indispensable brindar un valor agregado al bien o servicio que se prestara de esta manera se puede generar una percepción positiva en el usuario que va a traducirse en la fidelidad con la institución y que al mismo tiempo generara que esta se pueda diferenciar de las otras empresas que son su competencia (Valencia-Naranjo, 


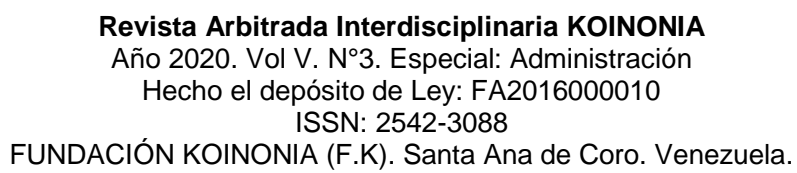

Verónica Alexandra Bernal-Rodas; Moisés Marcelo Matovelle-Romo; Cristina Guadalupe Ordoñez-Espinoza; Magdalena Emilia Ordoñez-Gavilanes

et al., 2019). Con relación a la satisfacción del usuario. Caldera, et al., (2011), manifiestan que esta figura está comprendida por tres elementos:

1. El rendimiento percibido: son aquellos que hacen relación al beneficio que el cliente considera haber conseguido después de adquirir un producto o servicio.

2. Las expectativas: se podría denominar aquella expectativa que tiene el usuario o cliente en cuanto al bien o servicio a recibir.

3. Los niveles de satisfacción: estos pueden ser; insatisfacción, satisfacción o complacencia.

A lo que refiere en líneas superiores podemos concluir que la satisfacción del cliente se encuentra íntimamente relacionado con el conjunto de emociones ya sean estas positivas o negativas que se producen en el cliente o usuario al momento de comparar el precio que ha tenido que pagar en un bien o servicio versus las expectativas que se tienen al momento de la adquisición, es decir si las emociones son positivas el cliente se sentirá satisfecho lo que a su vez se traducirá en la fidelidad del mismo con la institución y también la recomendación de este a futuros clientes, mientas si los sentimientos son negativos, es decir al cliente o usuario están insatisfechos no regresaran a la empresa, pasaran la voz en cuanto a su molestia y esto a su vez será una mala publicidad para la institución.

\section{La gestión de calidad y su importancia en el servicio al cliente o usuario en las Instituciones de Educación Superior}

La gestión de calidad se podría sintetizar en el conjunto de acciones o herramientas que utilizan las empresas o instituciones con la finalidad de evitar posibles errores en cuanto a los procesos de creación de sus productos o en el servicio que prestan, por esta razón se considera importante la implementación de un sistema que logre evaluar la gestión eficiente en cuanto a la calidad de una empresa que permitirá identificar los errores o debilidades para que de esta manera conjuntamente con su equipo de trabajo se creen 
Revista Arbitrada Interdisciplinaria KOINONIA

Año 2020. Vol V. №3. Especial: Administración

Hecho el depósito de Ley: FA2016000010

ISSN: 2542-3088

FUNDACIÓN KOINONIA (F.K). Santa Ana de Coro. Venezuela.

\section{Verónica Alexandra Bernal-Rodas; Moisés Marcelo Matovelle-Romo; Cristina Guadalupe Ordoñez-Espinoza; Magdalena Emilia Ordoñez-Gavilanes}

las soluciones y se logren solventar las dificultades con la finalidad de cambiar y mejorar el servicio o producto que se oferta (Argudo-Tello, et al., 2019).

Un buen sistema que permitirá medir la gestión de calidad de una institución debe contener las técnicas, herramientas y modelos necesarios para que una institución o empresa crezca a partir del reconocimiento de sus debilidades y fortalezas, así como los recursos técnicos y humanos necesarios para mejorar en cuanto a los productos 0 servicios que oferta, la clave para que un proceso de gestión de calidad sea efectivo en una empresa es crear un modelo que se adapte a las necesidades de la institución y que será flexible y adaptable a las necesidades de la empresa (Erazo-Álvarez \& NarváezZurita, 2020).

Por otro lado, el modelo de gestión como una herramienta que deberían tener las instituciones sobre el entorno dentro del cual va a realizar sus actividades, con la finalidad de comprenderlo, analizarlo y luego de esto si es necesario modificarlo, de esta manera se generará un diagnostico organizacional que le permita mejorar de forma continua y con la participación de todos quienes conforman la institución (Cubino, 2001).

De lo antes referido por Cubino debemos destacar que dentro de una institución se debe implementar un proceso de Gestión de Calidad, este proceso debe comenzar por una planificación dentro de la cual se definan las tareas de todos quienes conforman la institución, lo siguiente sería el desarrollo de esas tareas, es decir poner en ejecución de manera efectiva y eficaz las tareas que se han planificado, luego se tiene que comprobar, es decir hacer un contraste entre lo que se ha planificado y lo que se está desarrollando y por último se actúa, es decir se pone en marcha todos los procesos panificados y desarrollados con la finalidad de brindar un servicio o desarrollar un producto de calidad. Es importante insistir sobre la necesidad de un proceso adecuado para la implementación de herramientas para medir el grado de aceptación de las personas que recibirán un bien o servicio, esta gestión de calidad debe conjugar directamente con la pertinencia e impacto que se lograría tener en beneficio de la institución a la que se aplique, para lo 


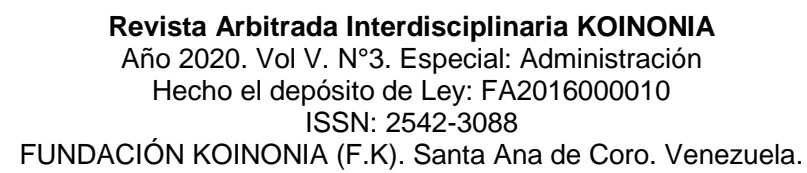

Verónica Alexandra Bernal-Rodas; Moisés Marcelo Matovelle-Romo; Cristina Guadalupe Ordoñez-Espinoza; Magdalena Emilia Ordoñez-Gavilanes

cual es importante contar con modelos que resulten exitosos en otras organizaciones para poder replicarlos adaptándolos a las realidades de cada empresa, Almada manifiesta que debido a la globalización y al gran desarrollo de propuestas educativas y de instituciones que brinden un servicio en el área educativa, se concibe la necesidad de que se implemente gestiones que puedan medir la calidad de los servicios que se presta sobre todo en la Educación Superior (Almada \& Troquet, 2009).

\section{Implementación de procesos de Gestión de Calidad que permitan desarrollar competencias para medir la satisfacción de los usuarios}

Dentro de la temática que nos ocupa que es la implementación de la Gestión de Calidad en los servicios prestados por las secretarias de las Unidades Académicas de la Universidad Católica de Cuenca, debemos destacar que en los últimos años las Instituciones de Educación Superior (IES), han venido preocupándose constantemente en cuanto a la calidad del servicio que brindan tanto a sus estudiantes como a la sociedad en general relacionado con la educación y a los servicios administrativos que ofrecen, de ahí entonces la importancia de implementar un proceso para poder medir la calidad del bien o servicio que a su vez le permita a la Universidad la identificación de cuáles son sus fortalezas y las debilidades que se tienen en los servicios que brindan a los estudiantes en las secretarias de las unidades académicas con la finalidad de crear los mecanismos necesarios para solventar deficiencias.

En las secretarias de las Unidades Académicas según el Reglamento de la Universidad Católica de Cuenca se concentran el mayor número de trámites administrativos; que los estudiantes de la Universidad ejecutan a lo largo de su carrera universitaria, así como las personas particulares que requieren ejecutar algún tipo de tramite relacionado con la Universidad, por ahí la importancia de la generación de un sistema de gestión de calidad, quien permita medir la satisfacción de los usuarios en cuanto al servicio que reciben dentro de estas dependencias. 


\section{Verónica Alexandra Bernal-Rodas; Moisés Marcelo Matovelle-Romo; Cristina Guadalupe Ordoñez-Espinoza; Magdalena Emilia Ordoñez-Gavilanes}

Como habíamos manifestado dentro de la gestión de calidad lo primero que se debe cumplir es la identificación de fortalezas y debilidades para lo cual es importante la aplicación de reactivos que permitan recolectar la información necesaria en cuanto a lo que establece, el cual permita contribuir a la imagen que se brinda sobre el modelo organizacional que se tiene en la universidad y la importancia de brindar la satisfacción y bienestar a los estudiantes y usuarios en general, en cuanto a la atención y servicios recibidos por el personal que labora dentro de las secretarias de las Unidades Académicas.

Los principios establecidos en la normativa interna de la Universidad direccionan a todos quienes forman parte de la institución a cumplir sus actividades de forma eficiente, implementando las herramientas necesarias para lograr este objetivo, comprometiéndose a mejorar constantemente con la finalidad de brindar un servicio apegado a los estándares de calidad para de esta manera crear en los estudiantes una percepción positiva en el área académica como administrativa, para esto es necesario el compromiso de las autoridades y del personal que labora en el área de secretaria para identificar y resolver las debilidades que hayan determinado en los procesos.

\section{Medición de la Satisfacción.}

Ahora bien, luego de haber tratado lo referente a lo que se considera usuario y satisfacción del mismo, se debe abordar como se puede medir esta satisfacción, uno de los modelos predilectos para la valoración es el diagrama causal mediante el cual se puede hacer una relación entre el servicio. 


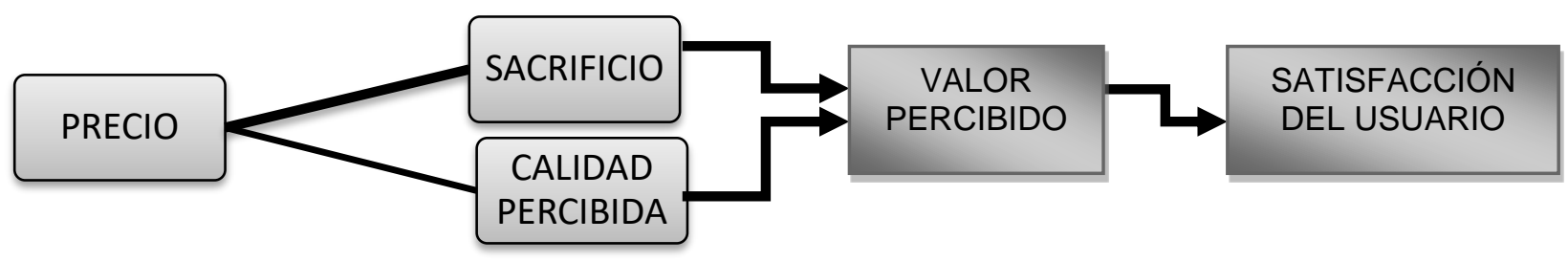

Figura 1. Modelo estructural de satisfacción. Fuente: (Dodds \& Monroe, 1985)

Modelos posteriores han utilizado esta figura como base para sus investigaciones posteriores donde se evalúan otros factores que caracterizan a un servicio, los cuales se comenzaron a evaluar cuantitativamente, lo cual además permitió innovar en lo referente a la validación de la satisfacción que el usuario percibe al momento de recibir un servicio determinado, un modelo de esta innovación se presenta en el modelo denominado SERVQUAL planteado por Parasuraman, donde la información que se evalúa es tomada directamente de los clientes antes y después de haber recibido el servicio, esta información es recopilada mediante encuestas estructuradas.

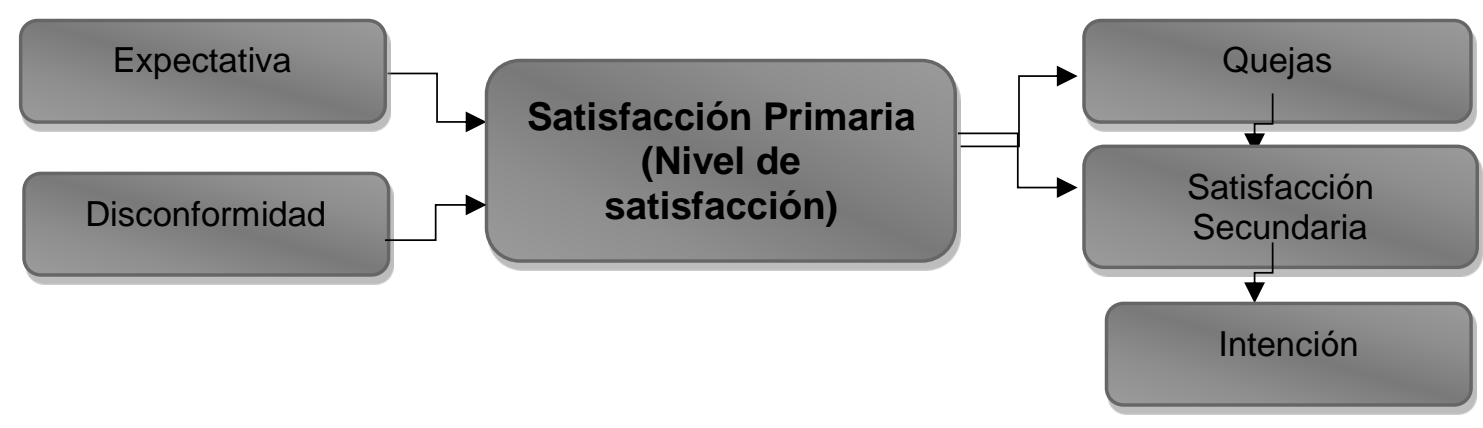

Figura 2. Modelo estructural SERVQUAL. Fuente: (Oliver, 1987). 
Revista Arbitrada Interdisciplinaria KOINONIA

Año 2020. Vol V. №3. Especial: Administración

Hecho el depósito de Ley: FA2016000010

ISSN: 2542-3088

FUNDACIÓN KOINONIA (F.K). Santa Ana de Coro. Venezuela.

\section{Verónica Alexandra Bernal-Rodas; Moisés Marcelo Matovelle-Romo; Cristina Guadalupe Ordoñez-Espinoza; Magdalena Emilia Ordoñez-Gavilanes}

Fue Feinberg quien a finales del año 90 permite evaluar la gestión en cuanto a la calidad incluye la intención de recomendar y recomprar el servicio-producto como medio valedero de evaluación, manifestando que la experiencia positiva demostrada por el cliente se ve reflejada en este tipo de acciones, de esta manera se puede determinar que el servicio al cliente se puede evaluar mediante un conjunto de factores y de aspectos relacionados con los clientes y no únicamente concentrarnos en el producto servicio prestado. La importancia de evaluar la satisfacción del cliente o usuario repercute necesariamente en la situación económica y competitiva de la institución o empresa, por esto la satisfacción del cliente ha tenido un alto interés tanto para directivos como para investigadores puesto como ya hemos visto la satisfacción se traduce en retención y esto a su vez está íntimamente ligado con la calidad, por lo cual como consecuencia de estas variables se crea la necesidad de establecer un proceso de evaluación en cuanto a la calidad. (Cordero, et al., 2019).

Para la investigación planteada evaluar la satisfacción de los usuarios que en su mayoría son los estudiantes de la Universidad Católica de Cuenca en cuanto a los servicios que reciben por parte del personal que labora en las secretarías de cada Unidad Académica, en los trámites administrativos que efectúan, se podrá determinar cuáles son las fortalezas que se tienen así como las debilidades en las cuales se tendrá que trabajar mediante la implementación de herramientas que permitirán mejorar la calidad del servicio que se presta, entendiendo que la evaluación de los procesos permite que las autoridades puedan identificar y trabajar en un mejoramiento constante en pro de la calidad universitaria, lo cual va a depender de varios factores como la capacitación, recursos adecuados, adquisición y desarrollo de competencias y el compromiso del personal (Mendieta-Ortega, et al., 2020).

Debemos considerar entonces que la identificación e implementación de competencias cumplan con la función de resolver con eficiencia cierto tipo de tareas ya sea que se tengan que dar en un contexto determinado, por lo cual es necesario establecer los 
Revista Arbitrada Interdisciplinaria KOINONIA

Año 2020. Vol V. №3. Especial: Administración

Hecho el depósito de Ley: FA2016000010

ISSN: 2542-3088

FUNDACIÓN KOINONIA (F.K). Santa Ana de Coro. Venezuela.

\begin{abstract}
Verónica Alexandra Bernal-Rodas; Moisés Marcelo Matovelle-Romo;
Cristina Guadalupe Ordoñez-Espinoza; Magdalena Emilia Ordoñez-Gavilanes
\end{abstract}

componentes que debemos tomar en cuenta al momento de medir la calidad, como son el valor que le damos al bien o servicio, si este cumple con el fin para el cual fue creado y si esto depende o no del carácter actitudinal de la partes involucradas (Vega-Monsalve, 2016).

Las gestiones administrativas en los procesos de Educación Superior han ido evolucionando por los avances tecnológicos y se han convertido en uno de los pilares fundamentales en cuanto a cómo el usuario en nuestro caso los estudiantes y las personas externas a la Universidad realizan diferentes trámites en las áreas administrativas como son las secretarías de las Unidades Académicas, dentro de las cuales se desarrollan varios procesos que deberían componerse de una serie de competencias, actitudes y aptitudes, las cuales permitan brindar una atención adecuada en la cual tanto la calidad, la calidez estén directamente implícitas (Lema-Guiracocha, et al., 2019).

\title{
METODOLOGÍA
}

Metodológicamente se basó en un tipo descriptivo no experimental transversal, con la finalidad de recopilar información mediante encuesta online y cuestionario de varias alternativas de respuesta en escala de Likert. La población para la presente investigación estuvo conformada por los presidentes (estudiantes) de cada curso de las Unidades Académicas de la Universidad Católica de Cuenca (ver tabla 1), con la finalidad de conocer su percepción y expectativas como usuarios de la gestión administrativa de la Universidad Católica de Cuenca. 
Revista Arbitrada Interdisciplinaria KOINONIA

Año 2020. Vol V. №3. Especial: Administración

Hecho el depósito de Ley: FA2016000010

ISSN: 2542-3088

FUNDACIÓN KOINONIA (F.K). Santa Ana de Coro. Venezuela.

Verónica Alexandra Bernal-Rodas; Moisés Marcelo Matovelle-Romo;

Cristina Guadalupe Ordoñez-Espinoza; Magdalena Emilia Ordoñez-Gavilanes

\section{Tabla 1}

Definición de la Población

Población

Cantidad

Unidad Académica de Salud y Bienestar 50

Unidad Académica de Ingeniería, Industria y 32

Construcción

Unidad Académica de Ciencias Sociales 37

Unidad Académica de Administración 20

Unidad Académica de Tecnologías de la 4

Información y Comunicación

Unidad Académica de Educación 5

Unidad Académica de Ciencias Agropecuarias 2

\begin{tabular}{ll}
\hline Total & 150
\end{tabular}


Verónica Alexandra Bernal-Rodas; Moisés Marcelo Matovelle-Romo;

Cristina Guadalupe Ordoñez-Espinoza; Magdalena Emilia Ordoñez-Gavilanes

Se procedió a calcular la muestra:

\title{
Calculo muestral
}

\section{Fórmula}

\author{
$\mathrm{n}=$ \\ $d 2{ }^{*}(N-1)+Z 2{ }^{*} p^{*} q$
}

$\mathrm{N}=150$ estudiantes

$$
\begin{aligned}
& Z=1,96 \\
& Z 2=3,84 \\
& p=0,05 \\
& q=0,95 \\
& d=0,1 \\
& d 2=0,01 \\
& p^{*} q=1,81 \\
& n=124 \text { estudiantes }
\end{aligned}
$$

Por consiguiente, la muestra encuestada, estuvo constituida por 124 estudiantes, procesándose la información recopilada, mediante estadística descriptiva. 
Revista Arbitrada Interdisciplinaria KOINONIA

Año 2020. Vol V. №3. Especial: Administración

Hecho el depósito de Ley: FA2016000010

ISSN: 2542-3088

FUNDACIÓN KOINONIA (F.K). Santa Ana de Coro. Venezuela.

Verónica Alexandra Bernal-Rodas; Moisés Marcelo Matovelle-Romo;

Cristina Guadalupe Ordoñez-Espinoza; Magdalena Emilia Ordoñez-Gavilanes

\section{RESULTADOS}

Para analizar la presente investigación se realizó la aplicación de encuestas, mismas que permitieron medir la evaluación de la calidad en el servicio recibido y la satisfacción de los usuarios. A continuación, se muestran las siguientes tablas 2 y 3 con la síntesis de los resultados obtenidos, mismas que se clasificaron según su dimensión de estudio:

\section{Tabla 2}

Resultados de la encuesta aplicada a la gestión de calidad

\begin{tabular}{|c|c|c|c|c|}
\hline \multirow[t]{2}{*}{ Dimensión } & \multirow[t]{2}{*}{ Pregunta } & \multicolumn{3}{|c|}{ Alternativas } \\
\hline & & $\mathrm{Si}$ & No & Desconozco \\
\hline Política & $\begin{array}{l}\text { ¿Existe algún tipo de política de gestión } \\
\text { de calidad en tu universidad? }\end{array}$ & 43 & 12 & 69 \\
\hline Gestión & $\begin{array}{l}\text { ¿Existe algún departamento o unidad } \\
\text { dedicado a ello en de tu universidad? }\end{array}$ & 51 & 10 & 63 \\
\hline Calidad & $\begin{array}{l}\text { En el caso de que no se haya } \\
\text { implantado ningún sistema de gestión } \\
\text { de calidad ¿conoce usted si está } \\
\text { previsto a corto plazo implantarlo? }\end{array}$ & 14 & 18 & 92 \\
\hline
\end{tabular}


Revista Arbitrada Interdisciplinaria KOINONIA

Año 2020. Vol V. №3. Especial: Administración

Hecho el depósito de Ley: FA2016000010

ISSN: 2542-3088

FUNDACIÓN KOINONIA (F.K). Santa Ana de Coro. Venezuela.

Verónica Alexandra Bernal-Rodas; Moisés Marcelo Matovelle-Romo;

Cristina Guadalupe Ordoñez-Espinoza; Magdalena Emilia Ordoñez-Gavilanes

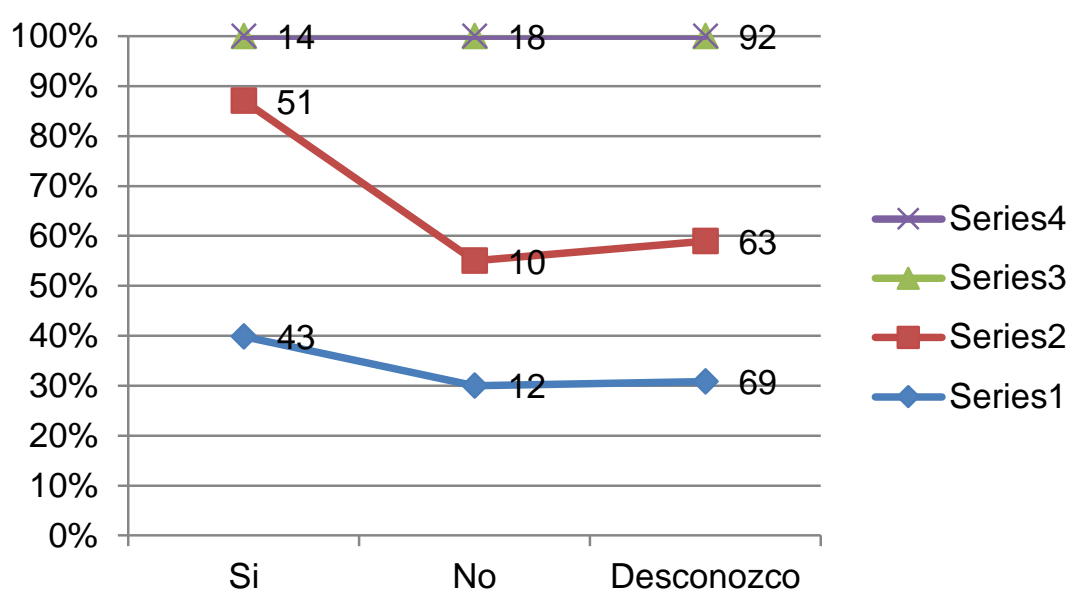

Figura 3. Resultados de la encuesta aplicada a la gestión de calidad

\section{Tabla 3}

Resultados de la encuesta aplicada para medir la satisfacción de los usuarios al momento de recibir el servicio

\begin{tabular}{lll} 
Pregunta & \multicolumn{2}{c}{ Alternativas } \\
& Excelente Bueno Malo Regular
\end{tabular}

¿Cuál es su grado de satisfacción con los servicios recibidos en la secretaria de su unidad académica?

¿Satisfacción de la secretaría de su unidad

¿Qué le parece el horario de atención en su unidad usuarios académica?

¿Considera adecuada la tecnología/medios con los que cuenta la universidad para solucionar cualquier incidencia que se da en la secretaria de su unidad académica? 
Verónica Alexandra Bernal-Rodas; Moisés Marcelo Matovelle-Romo;

Cristina Guadalupe Ordoñez-Espinoza; Magdalena Emilia Ordoñez-Gavilanes

¿Considera que la universidad recoge de manera adecuada sus quejas y sugerencias?

Preguntas

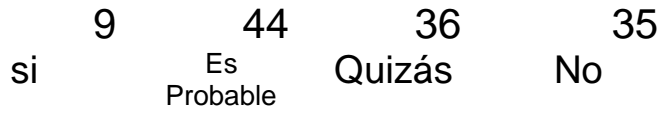

¿Considera que el personal que le atiende en la secretaria de su unidad académica le proporciona un servicio fiable Servicio y adecuado a sus necesidades?

25

61

25

27

28

29

40

¿Volvería a solicitar los servicios de la universidad? $\begin{array}{lll}41 & 38 & 32\end{array}$

13

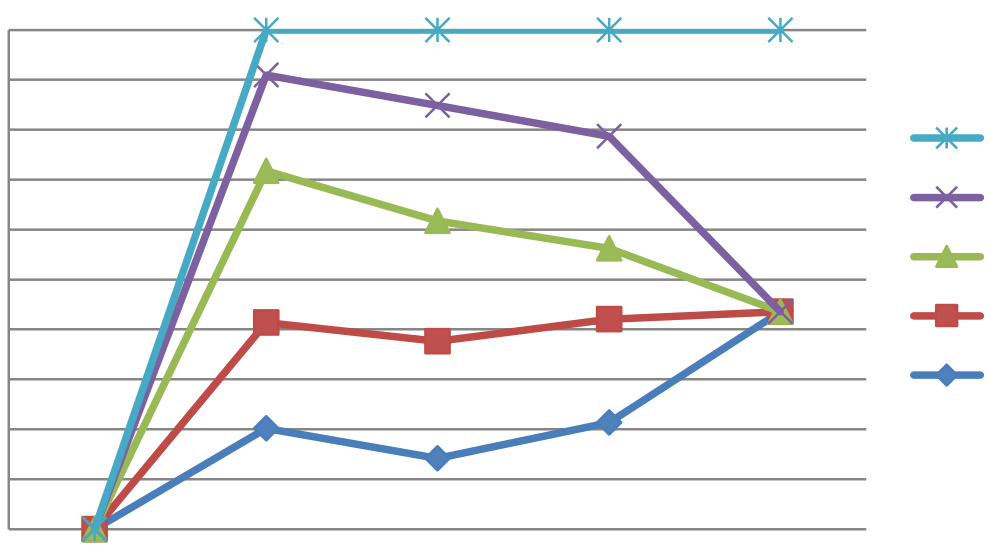

Figura 4. Resultados de la encuesta aplicada con respecto a la satisfacción de los usuarios. 
Verónica Alexandra Bernal-Rodas; Moisés Marcelo Matovelle-Romo; Cristina Guadalupe Ordoñez-Espinoza; Magdalena Emilia Ordoñez-Gavilanes

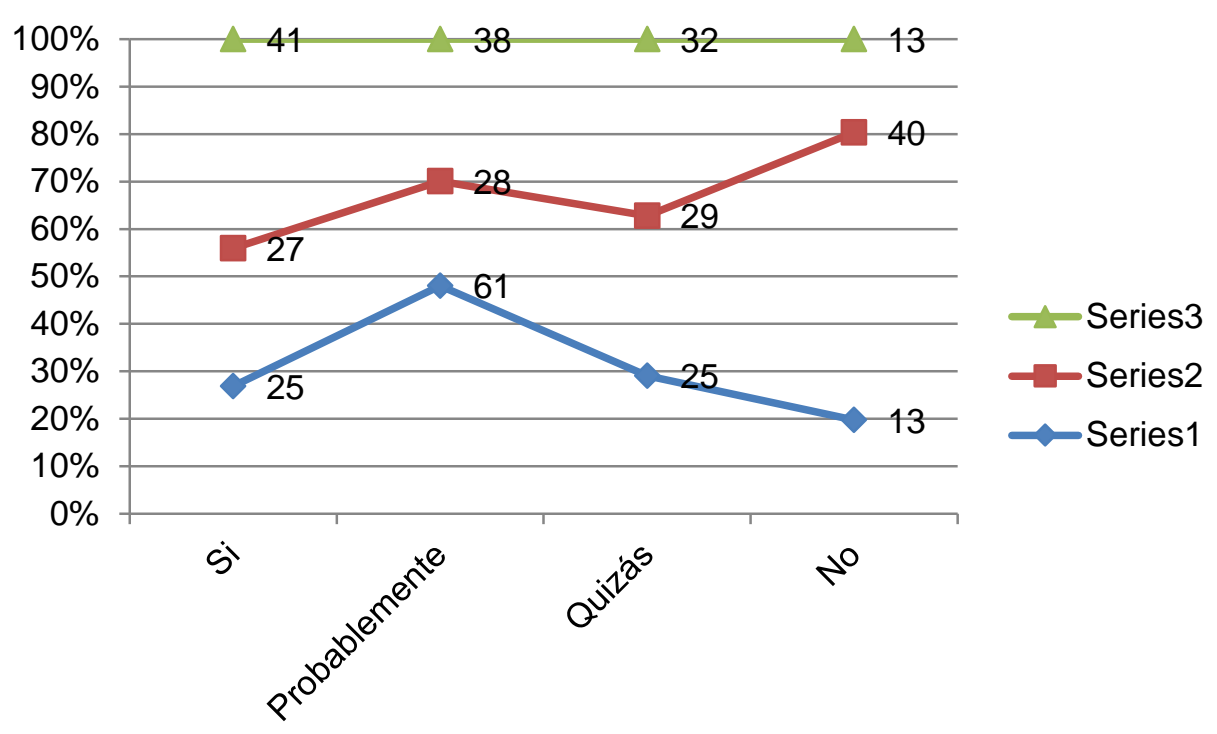

Figura 5. Resultados de la encuesta aplicada al momento de recibir el servicio

Con la información graficada y con los datos recolectados se puede analizar que el $60 \%$ de encuestados desconocen si la universidad cuenta con un sistema de gestión de calidad; así también se puede cotejar que al momento de medir la satisfacción de los servicios recibidos, la comunicación entre el personal, el horario de atención, la tecnología/medios para solucionar las incidencias y la manera de solventar adecuadamente las quejas y sugerencias en cada una de las unidades académicas por parte de la universidad tiene una aceptación del $47 \%$ por los usuarios y con relación a la información que proporciona el personal a los estudiantes, la recomendación del servicio y regresar a solicitar los servicios brindados por parte de la universidad el $34 \%$.

A continuación de haber realizado el análisis de cada una de las preguntas contestadas en la encuesta aplicada, se puede concluir que los resultados obtenidos reflejan que sería recomendable implementar un sistema de gestión de calidad (tecnológico) que permita calificar a los usuarios la satisfacción del servicio recibido en las secretarias de las Unidades Académicas de la Universidad Católica de Cuenca.

\section{PROPUESTA}


Verónica Alexandra Bernal-Rodas; Moisés Marcelo Matovelle-Romo; Cristina Guadalupe Ordoñez-Espinoza; Magdalena Emilia Ordoñez-Gavilanes

\section{Modelo de gestión de la calidad para la satisfacción de los usuarios}

Para la presente investigación se elabora el esquema de la propuesta basado en el modelo de gestión de la calidad, mismo que esta compuesto por los elementos que se detallan a continuación: planificación, control, aseguramiento de la calidad y la mejora continua, para conseguir la satisfacción de los usuarios al momento de recibir un servicio, dentro de las diferentes secretarias de las Unidades Académicas de la Universidad Católica de Cuenca.

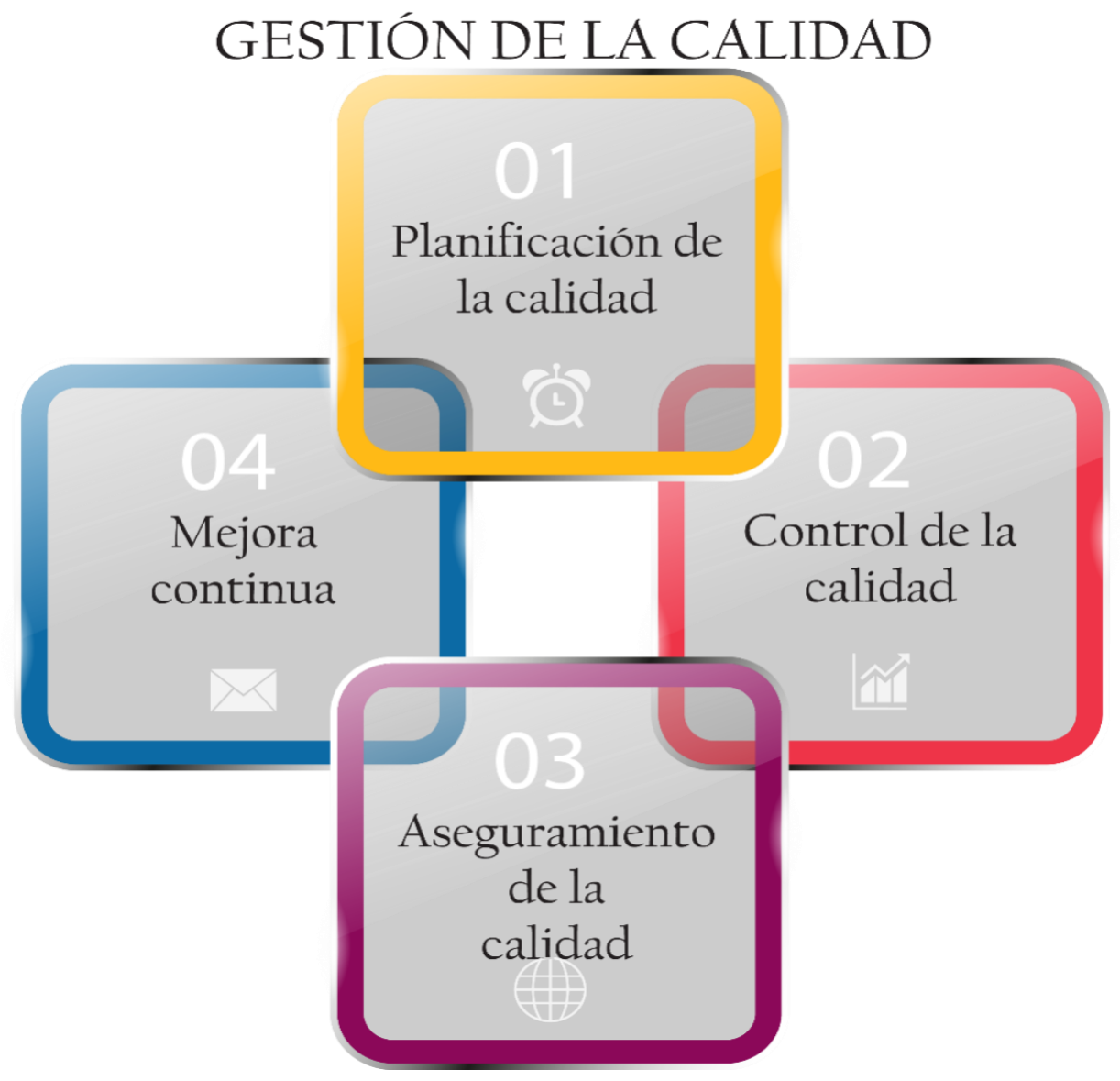

Figura 6. Esquema de la propuesta como modelo de gestión de la calidad.

\section{Planificación de la calidad}




\section{Verónica Alexandra Bernal-Rodas; Moisés Marcelo Matovelle-Romo; Cristina Guadalupe Ordoñez-Espinoza; Magdalena Emilia Ordoñez-Gavilanes}

Se propone implementar procesos para los servicios que se brindan a los estudiantes en las secretarias de las Unidades Académicas, para lo cual se debe comenzar identificando y determinando todos los pasos que sean necesarios para desarrollar y optimizar las actividades de estas dependencias, la finalidad de este proceso es contar con un manual estandarizado en donde conste los procesos y el control de los mismos.

Para la elaboración de la casa de la calidad QFD, se utilizaron los resultados obtenidos de las encuestas aplicadas a los estudiantes los cual ha permitido identificar las características del servicio y verificar si estas se ajustan a las necesidades de los usuarios, además nos permite observar cómo se encuentra la institución frente a las demás universidades y definir los aspectos primordiales que necesitan mejoras.

Se definen las características de calidad y las especificaciones del servicio recibido. Luego se procede a detallar los Qué's con los Cómo's; relacionando como se encuentra la institución frente a la competitividad y las características de calidad comparándolo con los de la competencia. Después se revisa la relación que tienen entre sí.

Convenciones utilizadas en la primera fase:

\section{RELACIONES}

$\begin{array}{cccc}\text { Fuerte } & 9 & 0 & \text { Minimizar } \\ \text { Moderada } & 3 & 0 & \text { Maximizar } \\ \text { Débil } & 1 & \triangle & \text { Objetivo }\end{array}$

Figura 7. Matriz QFD fase 1 y fase 2 
Revista Arbitrada Interdisciplinaria KOINONIA

Año 2020. Vol V. №3. Especial: Administración

Hecho el depósito de Ley: FA2016000010

ISSN: 2542-3088

FUNDACIÓN KOINONIA (F.K). Santa Ana de Coro. Venezuela.

Verónica Alexandra Bernal-Rodas; Moisés Marcelo Matovelle-Romo;

Cristina Guadalupe Ordoñez-Espinoza; Magdalena Emilia Ordoñez-Gavilanes

Al momento de emplear la metodología QFD, podemos observar ciertos beneficios dentro de la institución al momento de su aplicación como se detalla a continuación:

1. Integración de la calidad

2. Fijación de metas basadas en la cuantificación de las evaluaciones por parte de los usuarios

3. La planificación de mejorar el servicio resulta más específica

4. Las actividades de planificación y desarrollo están mas ligadas a las expectativas

5. Establece fuente de información para mejorar el servicio

6. Proporciona un sistema fiable de seguimiento del servicio a través del proceso

7. Jerarquiza las acciones de manera objetiva

8. Ayuda a la dirección a enfocar eficientemente sus esfuerzos

9. Reduce costes

10. Mayor satisfacción del cliente

11. Mayor transparencia en los procesos de desarrollo

12. Mejores relaciones entre los distintos servicios

13. Mayor reactividad

14. Mejora de la calidad y fiabilidad del servicio

Junto a estos beneficios del QFD, se pueden observar mejoras cuantificables basadas en el uso de la metodología por parte de las empresas, las mismas que se detallan en la figura que se detalla a continuación: 


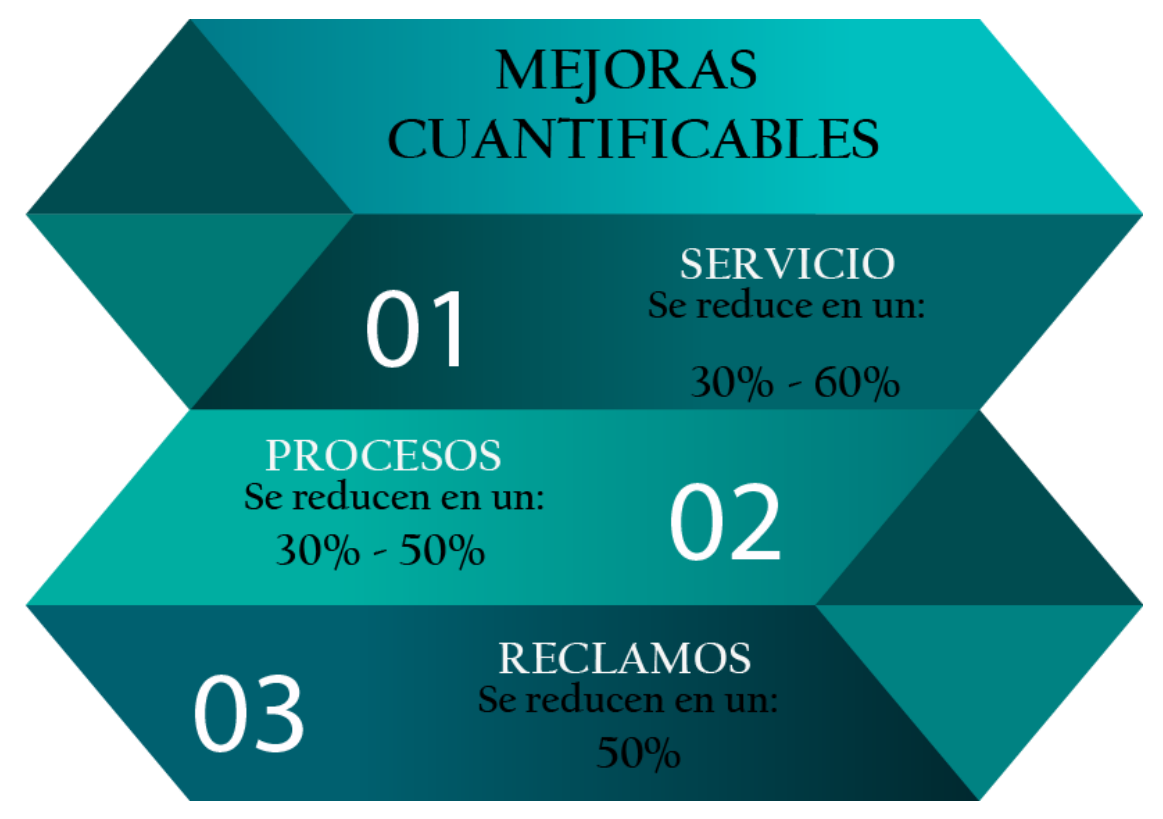

Figura 8. Mejoras cuantificables de las empresas basadas en el QFD

Sin embargo, no consiste en saber si el QFD permite reducir el ciclo de desarrollo de un servicio o cualquiera de las ventajas cuantitativas que se mencionaron anteriormente, sino se trata de conocer si el QFD permite aumentar la satisfacción del cliente y mejorar los resultados de la empresa.

\section{Control de la calidad}

Después de analizar los resultados obtenidos de los reactivos aplicados se propone la implementación de un sistema de gestión de calidad (tecnológico), mismo que permita a los usuarios calificar la atención del servicio recibido lo cual permite contar con una herramienta que permita tener un seguimiento detallado de los procesos que se manejan en las secretarias de las Unidades Académicas con la finalidad de mejorar la calidad del servicio que se ofrece a los estudiantes. 


\section{Verónica Alexandra Bernal-Rodas; Moisés Marcelo Matovelle-Romo; Cristina Guadalupe Ordoñez-Espinoza; Magdalena Emilia Ordoñez-Gavilanes}

El CWQC supone la integración de la calidad de la dirección, recursos humanos, operaciones, ambiente de trabajo, producto y servicio, la tabla que sigue recoge los siete aspectos que paulatinamente incorpora:

\section{Tabla 4}

Etapas de la implantación del CWQC, mediante la implementación de un sistema de gestión de calidad

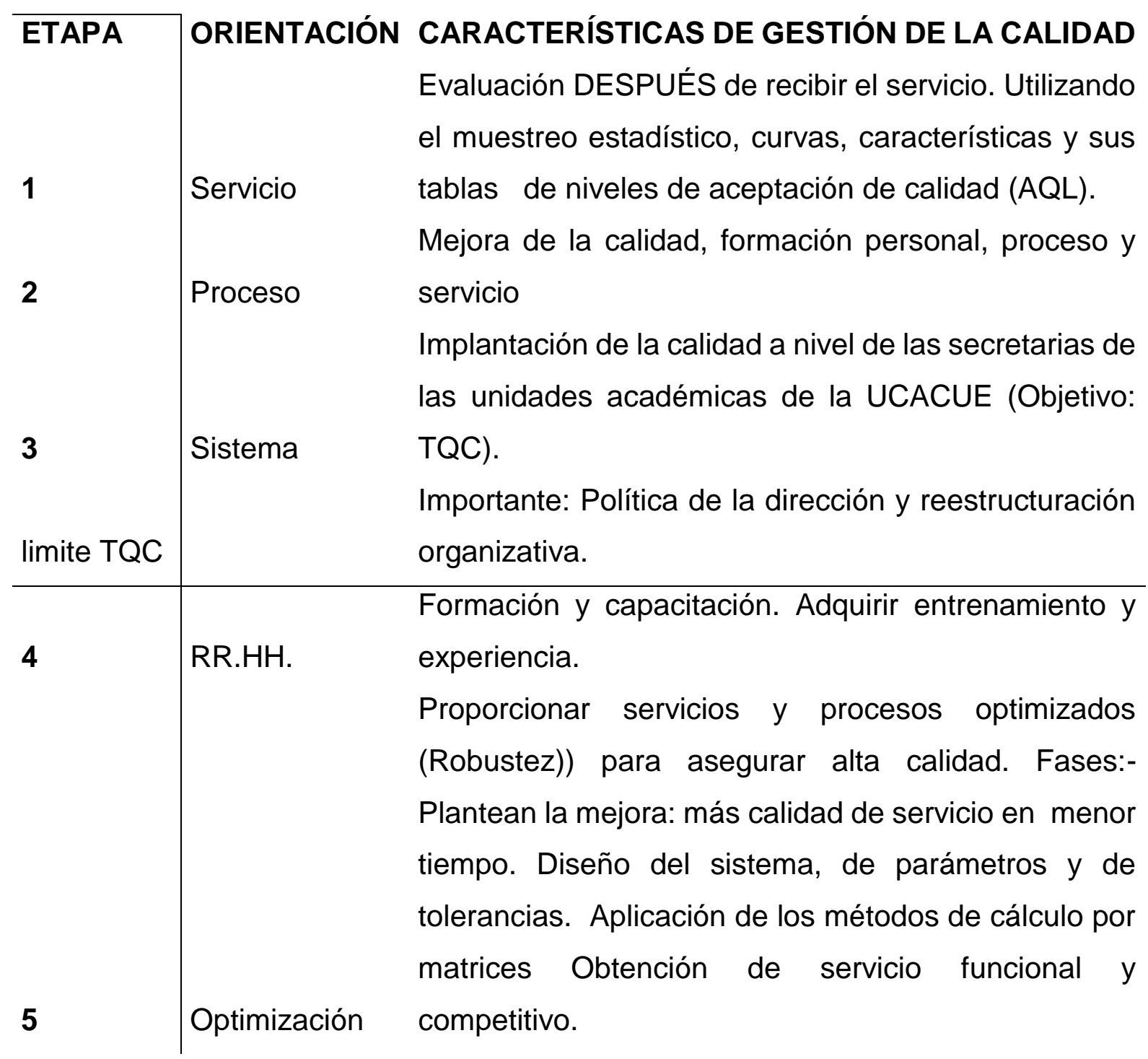


Verónica Alexandra Bernal-Rodas; Moisés Marcelo Matovelle-Romo; Cristina Guadalupe Ordoñez-Espinoza; Magdalena Emilia Ordoñez-Gavilanes

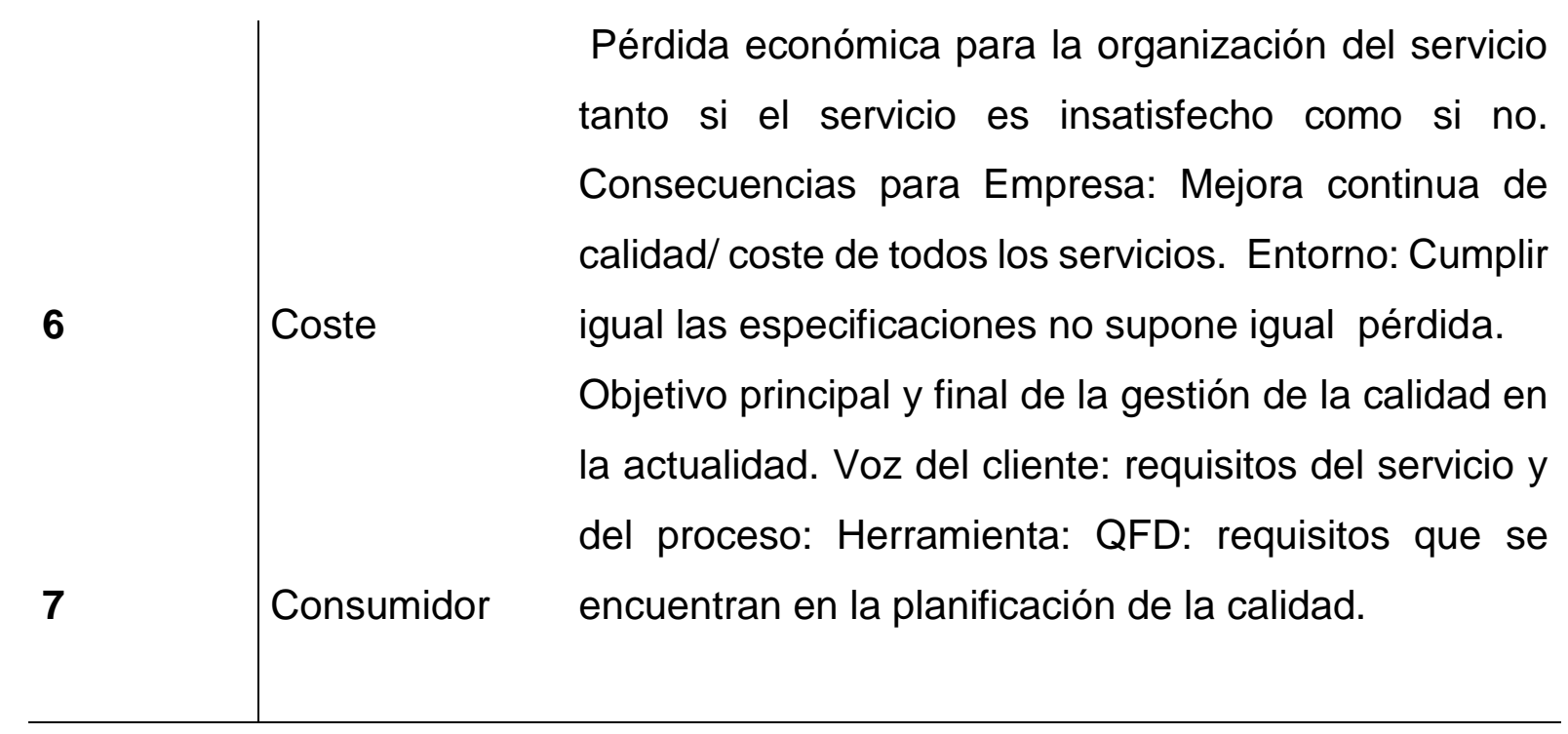

Se puede evidenciar, la calidad en la gestión de los recursos humanos, las herramientas de planificación y optimización, la preocupación por el coste, el consumidor y sus requerimientos como punto de arranque de la calidad. La calidad no es un proceso que se acaba cuando se alcanza un determinado nivel, sino que requiere una mejora y superación continua, pensando a mediano y largo plazo con el objeto de evolucionar constantemente.

\section{Aseguramiento de la calidad}

El aseguramiento de la calidad consiste en el proceso mediante el cual, luego de verificar los resultados obtenidos, mediante el control de calidad en relación a los servicios prestados por las secretarias de las Unidades Académicas se proceda a crear las herramientas necesarias para optimizar este servicio, dando seguimiento a las líneas de planificación establecidas dentro del sistema de gestión de calidad (tecnológico), con el fin de brindar a los estudiantes un servicio de calidad y así alcanzar la satisfacción de los usuarios. 
Revista Arbitrada Interdisciplinaria KOINONIA

Año 2020. Vol V. №3. Especial: Administración

Hecho el depósito de Ley: FA2016000010

ISSN: 2542-3088

FUNDACIÓN KOINONIA (F.K). Santa Ana de Coro. Venezuela.

Verónica Alexandra Bernal-Rodas; Moisés Marcelo Matovelle-Romo;

Cristina Guadalupe Ordoñez-Espinoza; Magdalena Emilia Ordoñez-Gavilanes

\section{Tabla 5}

Aseguramiento de la calidad para medir la satisfacción de los usuarios

\begin{tabular}{|c|c|c|c|}
\hline & Implementación & Impacto & Medición \\
\hline Áreas académicas & $\begin{array}{l}\text { Secretarias } \\
\text { unidades } \\
\text { académicas }\end{array}$ & $\begin{array}{l}\text { Mejoramiento } \\
\text { continuo }\end{array}$ & Auditorias semestrales \\
\hline Funcionarios & $\begin{array}{c}\text { Auxiliares de } \\
\text { secretaria }\end{array}$ & $\begin{array}{l}\text { Alto } \\
\text { rendimiento }\end{array}$ & $\begin{array}{c}\text { Sistema de evaluación } \\
\text { interno }\end{array}$ \\
\hline $\begin{array}{l}\text { Metodología, } \\
\text { procesos }\end{array}$ & $\begin{array}{l}\text { Manual de } \\
\text { funciones }\end{array}$ & $\begin{array}{l}\text { Conocimiento } \\
\text { del área de } \\
\text { desempeño }\end{array}$ & Satisfacción del usuario \\
\hline Herramientas & $\begin{array}{l}\text { Sistemas } \\
\text { operativos }\end{array}$ & $\begin{array}{c}\text { Menos } \\
\text { tiempo } \\
\text { operativo con } \\
\text { usuarios }\end{array}$ & $\begin{array}{l}\text { Sistema de gestión de } \\
\text { calidad }\end{array}$ \\
\hline Formatos & $\begin{array}{c}\text { Manuales } \\
\text { internos al } \\
\text { usuario }\end{array}$ & $\begin{array}{l}\text { Conocimiento } \\
\text { del manual }\end{array}$ & Incremento de usuarios \\
\hline
\end{tabular}

Para contar con un aseguramiento de la calidad continuo en las secretarias de las Unidades Académicas de la Universidad Católica de Cuenca, se propone implementar sistemas operativos de control que permitan a los distintos usuarios calificar el servicio 
recibido y que dicha información sirva como una herramienta de evaluación constante de los conocimientos del personal que labora en las áreas de las secretaria, estos insumos ayudaran a las autoridades a tomar decisiones a tiempo en cuanto al servicio brindado y de ser necesario modificar ciertos procesos con la finalidad de incrementar de manera eficiencia la experiencia de los usuarios que acuden a las secretarias, lo cual implica una mejora en los tiempos de despacho de requerimientos, logrando así conseguir un servicio de calidad y satisfacción de los usuarios.

\section{Mejora Continua}

Los procesos de mejora continua dentro de la Institución y en específico dentro de las secretarias de las Unidades Académicas, nos va a permitir conseguir resultados que se traduzcan en calidad en cuanto al servicio recibido por parte de los usuarios, el cual se obtendrá mediante un conjunto de acciones dirigidas a ofrecer un servicio eficaz mediante la implementación de un sistema de gestión de calidad adecuado y eficiente que permita identificar, definir, medir, analizar, mejorar y controlar procesos administrativos.

\section{Tabla 6}

La mejora continua, al momento de recibir el servicio 
Revista Arbitrada Interdisciplinaria KOINONIA

Año 2020. Vol V. №3. Especial: Administración

Hecho el depósito de Ley: FA2016000010

ISSN: 2542-3088

FUNDACIÓN KOINONIA (F.K). Santa Ana de Coro. Venezuela.

Verónica Alexandra Bernal-Rodas; Moisés Marcelo Matovelle-Romo;

Cristina Guadalupe Ordoñez-Espinoza; Magdalena Emilia Ordoñez-Gavilanes

\begin{tabular}{|c|c|c|c|c|}
\hline & Etapa & Conocimiento & Métodos & Medición \\
\hline 1 & Planificar & $\begin{array}{l}\text { Establecer } \\
\text { objetivos }\end{array}$ & Cuantitativos & $\begin{array}{c}\text { Medición de lo } \\
\text { planeado }\end{array}$ \\
\hline 2 & Realizar & $\begin{array}{c}\text { Puesta en } \\
\text { marcha }\end{array}$ & $\begin{array}{c}\text { Formación y } \\
\text { adecuación }\end{array}$ & Eficacia \\
\hline 3 & Comprobar & $\begin{array}{l}\text { Verificar y } \\
\text { controlar }\end{array}$ & $\begin{array}{l}\text { Cumplimiento } \\
\text { de objetivos }\end{array}$ & $\begin{array}{c}\text { Evaluación de } \\
\text { resultados }\end{array}$ \\
\hline 4 & Actuar & $\begin{array}{c}\text { Adecuación del } \\
\text { manual }\end{array}$ & $\begin{array}{c}\text { Adecuación del } \\
\text { proceso }\end{array}$ & $\begin{array}{l}\text { Medición de } \\
\text { resultado final }\end{array}$ \\
\hline
\end{tabular}

En esta primera etapa que es la de planificar se debe identificar claramente los objetivos que se quieren alcanzar y la elección de los métodos adecuados para lograrlos, en la segunda etapa que es la de realizar consiste en llevar a cabo las acciones correctivas planificadas en la fase anterior, la tercera fase es la que nos permite comprobar, verificar y controlar los efectos y resultados que surjan de la aplicación de estos procesos de las mejoras planificadas y la última fase es la de ejecución mediante la cual se demuestra que las acciones emprendidas han tenido los resultados esperados.

\section{Tabla 7}


Verónica Alexandra Bernal-Rodas; Moisés Marcelo Matovelle-Romo; Cristina Guadalupe Ordoñez-Espinoza; Magdalena Emilia Ordoñez-Gavilanes

Principales indicadores que se manejan en las secretarias de las unidades académicas de la UCACUE:

\begin{tabular}{|c|c|c|c|c|}
\hline No & \multicolumn{2}{|l|}{$\begin{array}{l}\text { Nombre del } \\
\text { indicador }\end{array}$} & Fórmula de Cálculo & Meta \\
\hline 1 & $\begin{array}{l}\text { Porcentaje } \\
\text { trámites } \\
\text { resueltos } \\
\text { secretaria }\end{array}$ & $\begin{array}{l}\text { de } \\
\text { en }\end{array}$ & $\begin{array}{l}=\text { (Número de trámites resueltos en secretaria) / (Número } \\
\text { de trámites que ingresan a secretaria) }{ }^{*} 100\end{array}$ & $90 \%$ \\
\hline 2 & $\begin{array}{l}\text { Porcentaje } \\
\text { registro } \\
\text { graduados en } \\
\text { carrera }\end{array}$ & $\begin{array}{l}\text { de } \\
\text { de } \\
\text { la }\end{array}$ & $\begin{array}{l}=(\text { Número de registros de graduados en la carrera) / } \\
\text { (Número de estudiantes que han presentado solicitud } \\
\text { para graduarse) } * 100\end{array}$ & $95 \%$ \\
\hline 3 & $\begin{array}{l}\text { Porcentaje } \\
\text { nivel } \\
\text { satisfacción } \\
\text { secretaria } \\
\text { los estudiant } \\
\text { de la unid } \\
\text { académica }\end{array}$ & $\begin{array}{l}\text { de } \\
\text { de } \\
\text { de } \\
\text { por } \\
\text { tes } \\
\text { dad }\end{array}$ & $\begin{array}{l}=\text { (Número de estudiantes que califican muy satisfactorio } \\
\text { y satisfactorio)/(Número de estudiantes que realizan } \\
\text { trámites en secretaria)* } 100\end{array}$ & $95 \%$ \\
\hline 4 & $\begin{array}{l}\text { Porcentaje } \\
\text { registros } \\
\text { calificaciones } \\
\text { recibidos } \\
\text { secretaria }\end{array}$ & $\begin{array}{l}\text { de } \\
\text { de } \\
\text { en }\end{array}$ & $\begin{array}{l}=(\text { Número de informes de registros de calificaciones } \\
\text { presentados }) /(\text { Número de asignaturas dictadas en el } \\
\text { semestre }) * 100\end{array}$ & $100 \%$ \\
\hline 5 & $\begin{array}{l}\text { Porcentaje } \\
\text { transferencia } \\
\text { documental }\end{array}$ & de & $\begin{array}{l}=(\text { Número de carpetas de titulación aprobadas } \\
\text { )/(Número de carpetas de titulación presentadas }) * 100\end{array}$ & $90 \%$ \\
\hline 6 & $\begin{array}{l}\text { Porcentaje } \\
\text { respuesta } \\
\text { peticiones } \\
\text { información }\end{array}$ & $\begin{array}{l}\text { de } \\
\text { de } \\
\text { de }\end{array}$ & $\begin{array}{l}=(\text { Número de solicitudes contestadas en menos de } 8 \\
\text { días }) /(\text { Número de solicitudes recibidas }) * 100\end{array}$ & $95 \%$ \\
\hline 7 & $\begin{array}{l}\text { Cumplimiento } \\
\text { Políticas } \\
\text { Normativas } \\
\text { Manejo } \\
\text { Archivo }\end{array}$ & $\begin{array}{l}\text { de } \\
\text { y } \\
- \\
\text { de }\end{array}$ & $\begin{array}{c}\text { Indicador de Cumplimiento (Tener reglamento de } \\
\text { políticas para secretaria y otros documentos } \\
\text { referentes) }\end{array}$ & \\
\hline
\end{tabular}


Revista Arbitrada Interdisciplinaria KOINONIA

Año 2020. Vol V. №3. Especial: Administración

Hecho el depósito de Ley: FA2016000010

FUNDACIÓN KOINONIA (F.K). Santa Ana de Coro. Venezuela.

Verónica Alexandra Bernal-Rodas; Moisés Marcelo Matovelle-Romo;

Cristina Guadalupe Ordoñez-Espinoza; Magdalena Emilia Ordoñez-Gavilanes

Porcentaje de trámites resueltos en secretaria

Nombre del

Indicador:

Tipo de Indicador:

Eficacia

Eficiencia

Efectividad

$\mathbf{X}$

Fórmula de $=$ (Número de trámites resueltos en secretaria) / (Número de trá

cálculo: que ingresan a secretaria) ${ }^{*} 100$

Meta:

$90 \%$

Bueno:

Aceptable:

Deficiente:

Rango de gestión:

Mayor o igual $90 \% \quad 89 \%-85 \%$

Menor o igual

$84 \%$

Periodicidad:

Semestral al finalizar el ciclo académico

Fuente:

- Secretaría de Carrera

- Solicitudes de faltas

- Solicitudes de trabajos y exámenes postergados

Evidencias:

- Solicitudes de supletorios

- Solicitudes de petición de record académico

- Solicitud de notas para becas 
Revista Arbitrada Interdisciplinaria KOINONIA

Año 2020. Vol V. №3. Especial: Administración

Hecho el depósito de Ley: FA2016000010

ISSN: 2542-3088

FUNDACIÓN KOINONIA (F.K). Santa Ana de Coro. Venezuela.

Verónica Alexandra Bernal-Rodas; Moisés Marcelo Matovelle-Romo;

Cristina Guadalupe Ordoñez-Espinoza; Magdalena Emilia Ordoñez-Gavilanes

Nombre del
Indicador:

Tipo de Indicador:

Eficacia $\quad$ Eficiencia $\quad$ Efectividad

$\mathbf{X}$

Fórmula de cálculo: $=($ Número de registros de graduados en la carrera)/(Númer estudiantes que $\mathrm{n}$ que han presentado solicitud para graduarse)*

Meta: $95 \%$

\section{Bueno:}

Rango de gestión:
Periodicidad:

Fuente:

Evidencias:

Mayor o igual a $95 \% \quad 94 \%-84 \%$

\section{Aceptable:}

Deficiente:

Menor o igual $83 \%$
Semestral al finalizar el ciclo académico

- Secretaría de Carrera

- Informe presentado por el responsable de seguimiento a graduados de cada carrera

- Solicitud presentada por el estudiante 
Verónica Alexandra Bernal-Rodas; Moisés Marcelo Matovelle-Romo; Cristina Guadalupe Ordoñez-Espinoza; Magdalena Emilia Ordoñez-Gavilanes

Nombre

del Porcentaje de nivel de satisfacción de secretaria por los Indicador: estudiantes de la unidad académica

\section{Tipo de Indicador:}

\section{Eficacia}

Eficiencia $\mathbf{X}$

Fórmula de $=$ (Número de estudiantes que califican muy satisfactorio y satisfac cálculo: / (Número de deestudiantes que realizan trámites en secretaria)* 1

Meta: $95 \%$

Rango de gestión:

Mayor o igual a $95 \% \quad 94 \%-80 \% \quad$ Menor o igual $79 \%$

Periodicidad: Semestral al finalizar el ciclo académico

Fuente:

- Secretaría de Carrera

Evidencias: momento de recibir el servicio 
Revista Arbitrada Interdisciplinaria KOINONIA

Año 2020. Vol V. №3. Especial: Administración

Hecho el depósito de Ley: FA2016000010

FUNDACIÓN KOINONIA (F.K). Santa Ana de Coro. Venezuela.

Verónica Alexandra Bernal-Rodas; Moisés Marcelo Matovelle-Romo;

Cristina Guadalupe Ordoñez-Espinoza; Magdalena Emilia Ordoñez-Gavilanes

Nombre

del Porcentaje de registro de calificaciones recibidos en secretaria

Indicador:

Tipo de Indicador:

Fórmula de cálculo:

Meta:

Rango de gestión:

Periodicidad:

Fuente:

Evidencias:

\section{Eficacia}

Eficiencia

$\mathbf{X}$

$=$ (Número de informes de registros de calificaciones presentad (Número de asignaturas dictadas dictadas en el semestre)* 100
Efectividad $100 \%$

Igual a $100 \% \quad 99 \%-95 \% \quad$ Menor o igual $94 \%$

Semestral al finalizar el ciclo académico

- Secretaría de Carrera

- Cuadro de calificaciones presentado por los docentes de cada carrera. 
Revista Arbitrada Interdisciplinaria KOINONIA

Año 2020. Vol V. №3. Especial: Administración

Hecho el depósito de Ley: FA2016000010

FUNDACIÓN KOINONIA (F.K). Santa Ana de Coro. Venezuela.

Verónica Alexandra Bernal-Rodas; Moisés Marcelo Matovelle-Romo;

Cristina Guadalupe Ordoñez-Espinoza; Magdalena Emilia Ordoñez-Gavilanes

Porcentaje de transferencia documental

Nombre del

Indicador:

Tipo de Indicador:

Eficacia

Eficiencia

Efectividad

$\mathbf{X}$

Fórmula de cálculo: =(Número de carpetas de titulación aprobadas ) / (Número de carpet titulación ntadas presentadas) $* 100$

Meta:

$90 \%$

Rango de gestión:

Bueno:

Aceptable:

Deficiente:

Mayor o igual a $90 \%$

$89 \%-75 \%$

Menor o igual $74 \%$

Periodicidad:

Semestral al finalizar el ciclo académico

Fuente:

- Secretaría de Carrera

- Registro de las carpetas presentadas en secretaria

Evidencias:

- Envió de documentación a colecturía para solicitar carpetas de los estudiantes

- Fiscalización de las carpetas enviadas a Secretaria General 
Revista Arbitrada Interdisciplinaria KOINONIA

Año 2020. Vol V. №3. Especial: Administración

Hecho el depósito de Ley: FA2016000010

ISSN: 2542-3088

FUNDACIÓN KOINONIA (F.K). Santa Ana de Coro. Venezuela.

Verónica Alexandra Bernal-Rodas; Moisés Marcelo Matovelle-Romo;

Cristina Guadalupe Ordoñez-Espinoza; Magdalena Emilia Ordoñez-Gavilanes

Nombre del Porcentaje de respuesta de peticiones de información

Indicador:

Tipo de Indicador:

Eficacia $\quad$ Eficiencia $\quad$ Efectividad

$\mathbf{X}$

Fórmula de cálculo:

$=($ Número de solicitudes contestadas en menos de 8 días ) / (Núme solicitudes recibidas) $* 100$

Meta:

$95 \%$

Rango de gestión:

Periodicidad:

Mayor o igual a $95 \% \quad 94 \%-88 \% \quad$ Menor o igual $87 \%$

Fuente:

Semestral al finalizar el ciclo académico

- Secretaría de Carrera

- Oficios recibidos por parte de las demás dependencias de la

Evidencias: UCACUE

- Oficios recibidos en general 
Revista Arbitrada Interdisciplinaria KOINONIA

Año 2020. Vol V. №3. Especial: Administración

Hecho el depósito de Ley: FA2016000010

ISSN: 2542-3088

FUNDACIÓN KOINONIA (F.K). Santa Ana de Coro. Venezuela.

Verónica Alexandra Bernal-Rodas; Moisés Marcelo Matovelle-Romo;

Cristina Guadalupe Ordoñez-Espinoza; Magdalena Emilia Ordoñez-Gavilanes

\begin{abstract}
Nombre del
Indicador:

Cumplimiento de Políticas y Normativas - Manejo de Archivo
\end{abstract}

Fórmula de cálculo:

Meta:

Periodicidad:

Fuente:

Evidencias:
No aplica

No aplica

No aplica

- Secretaría de Carrera

- Dirección de Carrera

- Secretaría General

- Manual de Archivo de Secretaria

- Modelo Pedagógico

- LOES

\title{
CONCLUSIONES
}

Como resultado relevante al momento de analizar el modelo de gestión de la calidad para la satisfacción de los usuarios, se puede observar que la calidad del servicio que reciben los estudiantes o usuarios al momento de recibir la atención en las secretarias de las unidades académicas no es totalmente satisfactoria.

También se puede observar mediante el análisis de los principales indicadores que se manejan dentro de las secretarias de las Unidades Académicas, que existen procesos sistemáticos los cuales se tienen que cumplir a través de la programación de metas, las cuales permitan llegar a un rango de gestión bueno y lograr cumplir con los indicadores en un $100 \%$.

Se recomienda la implementación de un sistema de gestión de calidad mismo que permitirá monitorear la planificación, control, mejoramiento y mejora continua de la calidad, para brindar un servicio de alta calidad. 
Revista Arbitrada Interdisciplinaria KOINONIA

Año 2020. Vol V. №3. Especial: Administración

Hecho el depósito de Ley: FA2016000010

ISSN: 2542-3088

FUNDACIÓN KOINONIA (F.K). Santa Ana de Coro. Venezuela.

Verónica Alexandra Bernal-Rodas; Moisés Marcelo Matovelle-Romo;

Cristina Guadalupe Ordoñez-Espinoza; Magdalena Emilia Ordoñez-Gavilanes

\section{FINANCIAMIENTO}

No monetario

\section{AGRADECIMIENTO}

A los estudiantes de las Unidades Académicas de la Universidad Católica de Cuenca; por el apoyo que prestaron en el desarrollo de la investigación.

\section{REFERENCIAS CONSULTADAS}

Almada, C., \& Troquet, M. (2009). Universidad: la calidad como herramienta de gestión [University: quality as a management tool]. Revista Iberoamericana de Educación, 49(1), 1-11.

Argudo-Tello, K. J., Erazo-Álvarez, J. C., \& Narváez-Zurita, C. I. (2019). Evaluación de Control Interno en Riesgos Estratégicos para la Dirección de Planificación de la Universidad de Cuenca. [Evaluation of Internal Control in Strategic Risks for the Planning Direction of the University of Cuenca]. Revista Arbitrada Interdisciplinaria Koinonía, 1(4), 67-96. http://dx.doi.org/10.35381/r.k.v4i1.372

Bernal-Ávila, E. M., Erazo-Álvarez, J. C., \& Narváez-Zurita, C. I. (2019). Estructuras organizativas favorables a la Eficiencia Empresarial. [Organizational structures favorable to Business Efficiency]. Revista Arbitrada Interdisciplinaria Koinonía, 4(1), 3-31. http://dx.doi.org/10.35381/r.k.v4i1.370

Caldera, E., Pirela, J., \& Ortega, E. (2011). Dimensiones para el estudio de la calidad de servicios bibliotecarios [Dimensions for the study of the quality of library services]. Recuperado de https://n9.cl/sy148

Cordero, J., Narváez, C., \& Erazo, J. (2019). La evaluación por competencias: una herramienta para determinar la productividad del talento humano. [Assessment by competencies: a tool to determine the productivity of human talent]. 593 Digital Publisher CEIT, 4(3-1), 76-96.

Cubino, R. (2001). Modelo Europeo de Excelencia [European Model of Excellence] recuperado de https://url2.cl/5x4SR 


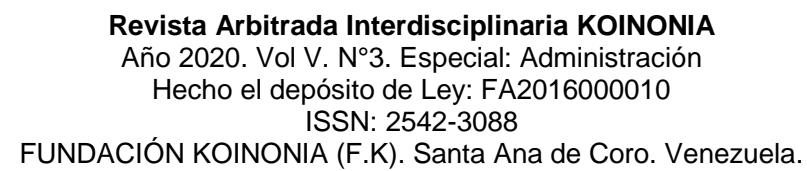

\begin{abstract}
Verónica Alexandra Bernal-Rodas; Moisés Marcelo Matovelle-Romo; Cristina Guadalupe Ordoñez-Espinoza; Magdalena Emilia Ordoñez-Gavilanes
\end{abstract}

Cuello-Cojitambo, J. D., Erazo-Álvarez, J. C., \& Narváez-Zurita, C. I. (2020). Visión sistémica de la gestión del conocimiento en el sector financiero cooperativo [Systemic vision of knowledge management in the cooperative financial sector]. Revista Arbitrada Interdisciplinaria Koinonía, 10(5), 607-637. http://dx.doi.org/10.35381/r.k.v5i10.707

Dodds, W., \& Monroe, K. (1985). The Effect of Brand and Price Information on Subjective Product Evaluations, in NA - Advances in Consumer Research Volume 12, eds. Elizabeth C. Hirschman and Moris B. Holbrook, Provo, UT: Association for Consumer Research, p. 85-90.

Erazo-Álvarez, J. C., \& Narváez-Zurita, C. I. (2020). Medición y gestión del capital intelectual en la industria del cuero - calzado en Ecuador. [Measurement and management of intellectual capital in the leather industry - footwear in Ecuador]. Revista Arbitrada Interdisciplinaria Koinonía, 9(5), 437-467. http://dx.doi.org/10.35381/r.k.v5i9.662

Lema-Guiracocha, P. E., Erazo-Álvarez, J. C., \& Narváez-Zurita, C. I. (2019). El talento humano, factor clave para la gestión organizacional en Instituciones de intermediación financiera. [Human talent, a key factor for organizational management in financial intermediation institutions]. Revista Arbitrada Interdisciplinaria Koinonía, 4(1), 349-375. http://dx.doi.org/10.35381/r.k.v4i1.461

Matsumoto-Nishizawa, R. (2014). Desarrollo del Modelo Servqual para la medición de la calidad del servicio en la empresa de publicidad Ayuda Experto. [Development of the Servqual Model for measuring the quality of service in the advertising company Expert Help]. PERSPECTIVAS, (34),181-209.

Mendieta-Ortega, M. P., Erazo-Álvarez, J. C., \& Narváez-Zurita, C. I. (2020). Gestión por competencias: herramienta clave para el rendimiento laboral del talento humano del sector hospitalario [Management by competences: key tool for the labor performance of the human talent of the hospital sector]. Revista Arbitrada Interdisciplinaria Koinonía, 10(5), 287-312. http://dx.doi.org/10.35381/r.k.v5i10.696

Oliver, R. L. (1987). An investigation of the interrelationship between consumer (dis)satisfaction and complaint reports. Advances in Consumer Research, 14(1), 218-222.

Valencia-Naranjo, D. E., Erazo-Álvarez, J. C., \& Narváez-Zurita, C. I. (2019). El clima organizacional y su incidencia en la motivación del Talento Humano. [The 
Revista Arbitrada Interdisciplinaria KOINONIA

Año 2020. Vol V. №3. Especial: Administración

Hecho el depósito de Ley: FA2016000010

ISSN: 2542-3088

FUNDACIÓN KOINONIA (F.K). Santa Ana de Coro. Venezuela.

Verónica Alexandra Bernal-Rodas; Moisés Marcelo Matovelle-Romo;

Cristina Guadalupe Ordoñez-Espinoza; Magdalena Emilia Ordoñez-Gavilanes

organizational climate and its impact on the motivation of Human Talent]. Revista Arbitrada Interdisciplinaria Koinonía, 1(4), 436-467. http://dx.doi.org/10.35381/r.k.v4i1.464

Vega-Monsalve, N. (2016). Neocompetencias, nuevo enfoque de competencias laborales en Salud Ocupacional. [Neocompetition, new approach to labor competition in occupational health]. Educación Médica Superior, 30(3), 627-638.

C2020 por los autores. Este artículo es de acceso abierto y distribuido según los términos y condiciones de la licencia Creative Commons Atribución-NoComercial-Compartirlgual 4.0 Internacional (CC BY-NC-SA 4.0)

(https://creativecommons.org/licenses/by-nc-sa/4.0/). 